ArTur Sowa (Saskatoon)

\title{
ON THE DIRICHLET MATRIX OPERATORS IN SEQUENCE SPACES
}

Abstract. We study the analytic properties of certain special operators, referred to as D-matrix operators, which arise naturally from classical Dirichlet series. There are a number of incentives for this work, including the applicability of D-matrix operators to signal processing via fast computational algorithms. It was observed in a prior publication that certain types of D-matrix operators are continuous in $\ell_{2}$ (Sowa 2013). In this work the focus is on a complementary case that arises in relation to the special D-matrix associated with Riemann's zeta function, and on its continuity properties in suitable Hilbert and Banach sequence spaces.

1. Introduction. We examine infinite matrices that arise from Dirichlet series, which we will refer to as Dirichlet matrices (D-matrices for short). More specifically, we consider the action of D-matrices in suitable Hilbert and Banach spaces. There are a number of incentives to study these linear operations. First, D-matrices are used as effective signal processing tools [14], [16], and arise naturally in the analysis of nonlinear oscillators [15]. Second, they are the basic tool in applications of the newly discovered phenomenon of broadband redundancy [19], in particular in its quantummechanical applications [20]. Third, their finite-dimensional reductions turn out to furnish the universal building blocks of generic matrices [17] -an observation that one may hope to generalize to infinite dimensions once the topological properties of infinite D-matrices are well understood.

A few fundamental results on the action of a class of D-matrices in $\ell_{2}$ are given in [18. However, the approach taken therein does not pro-

2010 Mathematics Subject Classification: 42C20, 47B99, 65T99, 65R32.

Key words and phrases: transforms for generalized harmonic analysis, Banach and Hilbert space operators, Dirichlet matrices, Dirichlet convolution.

Received 4 January 2017.

Published online 7 July 2017. 
vide any information about the special case that is related to the series $\zeta(s)=\sum n^{-s}$ which defines Riemann's zeta function [8. A study of its properties is the focus of the present work, particularly Sections 2 and 3 . It is part of the folklore of applied mathematics that continuity properties of operators in normed spaces translate, although not always trivially, into the stability of their numerical implementations. Other properties, such as surjectivity of an operator acting in abstract spaces, may provide insight into the stability of the corresponding numerical inverse problem, etc. The present work highlights some of the theoretical tools that are indispensable in addressing problems of this kind in relation to the action of D-matrices.

Preexisting literature on the subject is rather scarce. Some related information may be found in [4] and [11. Also, some related constructions are found in the engineering literature [9], [10]. In the broader sense, however, the subject matter is related to the well studied themes of complete systems in the Banach and Hilbert spaces, particularly nonharmonic Fourier series [22] and wavelets [6], 21], as well as a slew of topics at the intersection of analytic number theory and harmonic analysis (see e.g. [13]).

2. Preliminaries. Let $s=\sigma+i t \in \mathbb{C}$, where $\sigma, t \in \mathbb{R}$. For a sequence of complex numbers $a_{1}, a_{2}, \ldots$ we define a family of infinite matrices parametrized by $s$ (denoted $A_{s}$ or $\left[\left(a_{n} n^{-s}\right)\right]$ ) by

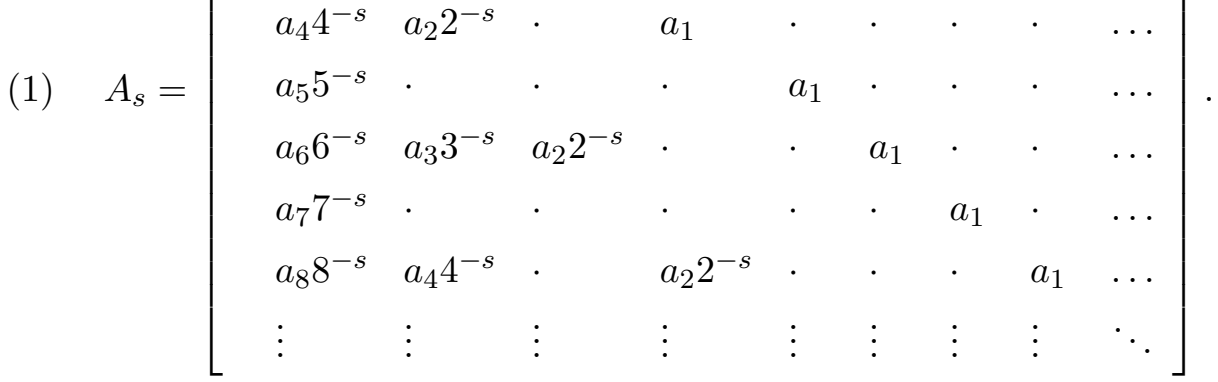

We will call matrices $A_{s}$ (with fixed $s \in \mathbb{C}$ ) Dirichlet matrices. For simplicity, we often write $A_{0}=A$. We say that a Dirichlet matrix $A_{s}$ is finitely supported if the sequence $\left(a_{n}\right)$ has only finitely many nonzero elements. In parallel to the above we let $A(s)$ denote the Dirichlet series

$$
A(s)=a_{1}+a_{2} 2^{-s}+a_{3} 3^{-s}+a_{4} 4^{-s}+\cdots .
$$


We will also make frequent use of the following notation:

$$
A^{\#}(s)=\left|a_{1}\right|+\left|a_{2}\right| 2^{-s}+\left|a_{3}\right| 3^{-s}+\left|a_{4}\right| 4^{-s}+\cdots .
$$

Throughout the article, $\sigma_{\mathrm{a}}(A)$ denotes the abscissa of absolute convergence of $A(s)$, i.e. the smallest real number with the property that $A^{\#}(\sigma)$ converges for all $\sigma>\sigma_{\mathrm{a}}(A)$.

It was demonstrated in [18] that the Dirichlet matrices (with $s=0$ ) form a ring. Moreover, the identification

$$
A \leftrightarrow A(s)
$$

provides an isomorphism of this ring with the ring of formal Dirichlet series $\left({ }^{1}\right)$. Since $A \leftrightarrow A_{s}$ is obviously a ring isomorphism, so also is

$$
A_{s} \leftrightarrow A(s) .
$$

Throughout the article we will pay special attention to the matrix that comes from constant sequence, i.e. $Z=[(1,1,1, \ldots)]$ (and also to $\left.Z_{s}\right)$. We note that the formal inverse $Z_{s}^{-1}$ equals $M_{s}=\left[\left(\mu(n) n^{-s}\right)\right]$, where $\mu$ is the Möbius function, i.e. $\mu(1)=1, \mu(n)=(-1)^{r}$ if $n$ is a product of $r$ distinct primes, and $\mu(n)=0$ otherwise. Of course, $Z(s)=\zeta(s)$, where $\zeta$ is Riemann's zeta function, and $M(s)=1 / \zeta(s)$.

Let $f: \mathbb{N} \rightarrow \mathbb{C}$ be a sequence. We let $A[f]$ denote the product of the matrix $A$ and the column vector $[f(1), f(2), \ldots]^{T}$. When convenient, the column vector $A[f]$ will be identified with the sequence $A[f]: \mathbb{N} \rightarrow \mathbb{C}$. Let us observe

$$
A_{s}[f](n)=\sum_{d \mid n} f\left(\frac{n}{d}\right) a_{d} d^{-s} \quad(\text { summation over all divisors of } n) .
$$

Also,

$$
A_{s}^{T}[f](n)=\sum_{k=1}^{\infty} f(n k) a_{k} k^{-s} .
$$

In what follows we will make frequent use of the special sequences $X_{r}: \mathbb{N} \rightarrow \mathbb{C}$ defined by $X_{r}(n)=n^{-r}$, where $r \in \mathbb{C}$ is a parameter. A direct calculation reveals the following interesting identity:

$$
A_{s}^{T} X_{r}=A(r+s) X_{r} .
$$

This means that each $X_{r}$ ia a formal eigenvector of $A_{s}^{T}$. Of course for the right-hand side to make sense, it is necessary that $A(r+s)$ converges, e.g. $\Re(r+s)>\sigma_{\mathrm{a}}(A)$.

$\left({ }^{1}\right)$ That is, the ring of all Dirichlet series (whether or not convergent anywhere in the complex plane) with addition given by $\sum a_{n} n^{-s}+\sum b_{n} n^{-s}=\sum\left(a_{n}+b_{n}\right) n^{-s}$ and multiplication given by $\sum a_{n} n^{-s} \cdot \sum b_{n} n^{-s}=\sum c_{n} n^{-s}$ with $c_{n}=\sum_{d \mid n} a_{d} b_{n / d}$. For more information on the Dirichlet ring see e.g. [2] or [3]. 
On the other hand, matrices of the form $A$ (or $A_{r}$ ) typically admit fewer nontrivial (formal) eigenvectors, e.g. we observe that

$$
Z f=\lambda f \text { implies } f=0 .
$$

Indeed, assume first $\lambda \neq 1$. Then $Z[f](1)=f(1)=\lambda f(1)$ implies $f(1)=0$. Next, we observe $Z[f](2)=0+f(2)=\lambda f(2)$, i.e. $f(2)=0$, etc., with the conclusion that $f \equiv 0$. If on the other hand $\lambda=1$, we have $Z[f](2)=$ $f(1)+f(2)=f(2)$, so $f(1)=0$. Now, let us assume that $k$ is the smallest integer for which $f(k) \neq 0$. It follows that $Z[f](2 k)=f(k)+f(2 k)=f(2 k)$, which is a contradiction, so that again $f \equiv 0$.

The elementary observations expressed in (2) and (3) motivate the systematic investigation of spectra of Dirichlet operators, which is the focus of this article.

3. Main observations. We wish to consider matrices of the form $A_{s}$ or $A_{s}^{T}$ as operators acting in topological linear spaces. Identifying the suitable spaces, i.e. spaces that make the analysis of such operators interesting and technically feasible, is part of the challenge. As we will see, it is natural to consider a particular set of weighted Hilbert spaces of sequences. We also consider the most interesting special matrices $Z$ and $Z^{T}$ as operators on the Banach space $\ell_{1}$ as well as on weighted spaces $\ell_{1, \mathrm{~m}}$ and $\ell_{\infty, \mathrm{m}^{-1}}$ with a suitably defined measure $m$. The elementary observations given above supply helpful preliminary insights into the matter, and will be further exploited in what follows.

3.1. $A_{s}$ and $A_{s}^{T}$ as operators in a weighted Hilbert space. We select the domain and target spaces for $Z$ and $Z^{T}$ so as to ensure that the resulting operators are bounded. We first examine a weighted Hilbert space reminiscent of the Sobolev spaces. Namely, for $p \in \mathbb{R}$ let us define

$$
\mathrm{h}^{p}=\left\{f: \mathbb{N} \rightarrow \mathbb{C}:\|f\|_{p}:=\left(\sum_{n \in \mathbb{N}}|f(n)|^{2} n^{2 p}\right)^{1 / 2}<\infty\right\} .
$$

The following facts are easily established by standard arguments:

- The formula $\langle f \mid g\rangle=\sum_{n} f(n) \overline{g(n)} n^{2 p}$ prescribes a sesquilinear form in $\mathrm{h}^{p}$. The collection of vectors $e_{n}=n^{-p} \delta_{n}$ furnishes an orthonormal basis in $\mathrm{h}^{p}$. Furthermore, this basis furnishes an isomorphism $\mathrm{h}^{p} \leftrightarrow \ell_{2}$. In particular, $\mathrm{h}^{p}$ is a (complete) Hilbert space.

- The dual space of $\mathrm{h}^{p}$ is $\left(\mathrm{h}^{p}\right)^{*}=\mathrm{h}^{-p}$.

- The following analogue of the Rellich Compactness Theorem holds: If $p>q$, then the natural inclusion $I: \mathrm{h}^{p} \hookrightarrow \mathrm{h}^{q}$ is a compact operator. Indeed, let $I_{N}$ be the finite rank operator given by $I_{N}[f](n)=f(n)$ for $n \leq N$, and $I_{N}[f](n)=0$ for $n>N$. It is easily seen that $\left\|I-I_{N}\right\|_{\mathrm{h}^{p} \rightarrow \mathrm{h}^{q}} \rightarrow 0$ as $N \rightarrow \infty$. 
We let $A_{s}: \mathrm{h}^{p} \rightarrow \mathrm{h}^{p}$ (resp. $A_{s}^{T}: \mathrm{h}^{p} \rightarrow \mathrm{h}^{p}$ ) signify the linear operator determined by the action of the matrix (1) (resp. its transpose) on $\mathrm{h}^{p}$. Note that the matrix $A_{s}^{T}$ represents the operator $A_{s}^{T}: \mathrm{h}^{p} \rightarrow \mathrm{h}^{p}$ in the formal basis $\left\{\delta_{n}\right\}$. It is useful to observe the following

Lemma 3.1. Let $\tilde{A}_{s}^{T}$ denote the matrix of the operator $A_{s}^{T}: \mathrm{h}^{p} \rightarrow \mathrm{h}^{p}$ in the basis $\left\{e_{n}\right\}$. Then

$$
\tilde{A}_{s}^{T}=A_{s+p}^{T}
$$

Proof. We have

$$
\begin{aligned}
A_{s}^{T} e_{n} & =n^{-p} A_{s}^{T} \delta_{n}=n^{-p} \sum_{d \mid n} a_{d} d^{-s} \delta_{n / d} \\
& =n^{-p} \sum_{d \mid n} a_{d} d^{-s}\left(\frac{n}{d}\right)^{p} e_{n / d}=\sum_{d \mid n} a_{d} d^{-(s+p)} e_{n / d} .
\end{aligned}
$$

Thus, the $(m, n)$ entry of the matrix $\tilde{A}_{s}^{T}$ can be nonzero only if $m \mid n$, in which case it is equal to $a_{n / m}(n / m)^{-(s+p)}$. This proves the claim.

In what follows we let $\Sigma_{p}(T)$ denote the spectrum of an operator $T$ : $\mathrm{h}^{p} \rightarrow \mathrm{h}^{p}$.

Proposition 3.1. Fix $p \in \mathbb{R}$ and consider $A_{s}^{T}: \mathrm{h}^{p} \rightarrow \mathrm{h}^{p}$ where $s=$ $\sigma+i t$. Assuming $\sigma>\sigma_{\mathrm{a}}(A)-p$, we have the following facts:

(i) $A_{s}^{T}$ is bounded, and $\left\|A_{s}^{T}\right\| \leq A^{\#}(p+\sigma)$.

(ii) $\Sigma_{p}\left(A_{s}^{T}\right) \supseteq\left\{a_{1}\right\} \cup\{A(r+s): r \in \mathbb{C}, \Re r>p+1 / 2\}$.

(iii) $A_{s}=\left(A_{s}^{T}\right)^{*}: \mathrm{h}^{-p} \rightarrow \mathrm{h}^{-p}$ is bounded with $\left\|A_{s}\right\| \leq A^{\#}(p+\sigma)$, and

$$
\Sigma_{-p}\left(A_{s}\right) \supseteq\left\{\bar{a}_{1}\right\} \cup\{\overline{A(r+s)}: r \in \mathbb{C}, \Re r>p+1 / 2\} .
$$

Proof. (i) In order to highlight the broader ramifications of this claim we provide two different proofs. The first proof makes use of Lemma 3.1 Observe that the $\ell_{1}$ norms of all the rows and columns of the matrix $\dot{A}_{s}^{T}$ are bounded above by $A^{\#}(p+\sigma)$. It is a known general fact $\left[\left(^{2}\right)\right.$ that this implies boundedness of the operator in $\ell_{2}$, and

$$
\left\|\tilde{A}_{s}^{T}\right\|_{\ell_{2} \rightarrow \ell_{2}} \leq A^{\#}(p+\sigma) \text {. }
$$

Since $\left\|\tilde{A}_{s}^{T}\right\|_{\ell_{2} \rightarrow \ell_{2}}=\left\|A_{s}^{T}\right\|_{\mathrm{h}^{p} \rightarrow \mathrm{h}^{p}}$, this completes the first proof.

The second proof is self-contained, and seems to shed more light on what is involved. Namely, let $g=A_{s}^{T} f$, where $s=\sigma+i t$, and let $p+\sigma>\sigma_{\mathrm{a}}(A)$.

$\left({ }^{2}\right)$ For a proof see [12, Chapter III, Example 2.3]. 
Observe that

$$
\begin{aligned}
\|g\|_{p}^{2} & =\sum_{m \in \mathbb{N}}|g(m)|^{2} m^{2 p}=\sum_{m \in \mathbb{N}} m^{2 p}\left|\sum_{n \in \mathbb{N}} f(m n) a_{n} n^{-s}\right|^{2} \\
& =\left.\left.\sum_{m \in \mathbb{N}} m^{2 p}\left|\sum_{n \in \mathbb{N}} f(m n) \operatorname{sgn}\left(a_{n}\right)\right| a_{n}\right|^{1 / 2} n^{-s / 2} n^{p / 2}\left|a_{n}\right|^{1 / 2} n^{-s / 2} n^{-p / 2}\right|^{2} \\
& \leq \sum_{m \in \mathbb{N}} m^{2 p} \sum_{n \in \mathbb{N}}|f(m n)|^{2}\left|a_{n}\right| n^{-\sigma} n^{p} \sum_{n \in \mathbb{N}}\left|a_{n}\right| n^{-(p+\sigma)} \quad \text { (by Cauchy-Schwarz) } \\
& =A^{\#}(p+\sigma) \sum_{m \in \mathbb{N}} \sum_{n \in \mathbb{N}}|f(m n)|^{2} m^{2 p} n^{2 p}\left|a_{n}\right| n^{-(p+\sigma)} \\
& =A^{\#}(p+\sigma) \sum_{n \in \mathbb{N}}\left|a_{n}\right| n^{-(p+\sigma)} \sum_{m \in \mathbb{N}}|f(m n)|^{2}(m n)^{2 p} \\
& \leq A^{\#}(p+\sigma)^{2}\|f\|_{p}^{2} .
\end{aligned}
$$

Note that in the penultimate line the order of summation is changed, which is allowed in a sum of nonnegative elements. This estimate shows that $\left\|A_{s}^{T}\right\|_{\mathrm{h}^{p} \rightarrow \mathrm{h}^{p}} \leq A^{\#}(p+\sigma)$.

(ii) We will use observation (2). Note that $X_{r} \in \mathrm{h}^{p}$ for all $r$ with $\Re r>p+1 / 2$. Also, $\Re r>p+1 / 2$ and $p+\sigma>\sigma_{\mathrm{a}}(A)$ imply $\Re(s+r)>$ $\sigma_{\mathrm{a}}(A)+1 / 2$. Therefore, in view of $(2)$, all elements of the set $\{A(r+s)$ : $r \in \mathbb{C}, \Re r>p+1 / 2\} \subseteq \mathbb{C}$ represent eigenvalues of $A_{s}^{T}$. In addition, observe $A_{s}^{T} e_{1}=a_{1} e_{1}$, i.e. $a_{1}$ is also an eigenvalue.

(iii) Let $u \in\left(\mathrm{h}^{p}\right)^{*}=\mathrm{h}^{-p}$ and $g \in \mathrm{h}^{p}$, and observe that

$$
\begin{aligned}
u\left[A_{s}^{T} g\right] & =\sum_{n=1}^{\infty} u(n) \sum_{k=1}^{\infty} g(n k) a_{k} k^{-s} \\
& =\sum_{m=1}^{\infty} g(m) \sum_{d \mid m} u(d) a_{m / d}\left(\frac{m}{d}\right)^{-s}=g\left[A_{s} u\right],
\end{aligned}
$$

and so $\left({ }^{3}\right) A_{s}=\left(A_{s}^{T}\right)^{*}$. This also implies that the norms of the two operators are equal. Since $\Sigma_{-p}\left(A_{s}\right)=\overline{\Sigma_{p}\left(A_{s}^{T}\right)}$, this proves the claim.

Corollary 3.1. If $A$ is finitely supported, say $a_{n}=0$ for all $n>N$, then $A_{s}^{T}: \mathrm{h}^{p} \rightarrow \mathrm{h}^{p}$ is bounded for all $s$ and all $p$, and $\left\|A_{s}^{T}\right\| \leq A^{\#}(p+\sigma)=$ $\sum_{n \leq N}\left|a_{n}\right| n^{-(p+\sigma)}$.

Proof. It suffices to repeat either one of the proofs of claim (i) in Proposition 3.1, and observe that the assumption $\sigma>-p+\sigma_{\mathrm{a}}(A)$ may be dropped. (In other words, one may assume $\sigma_{\mathrm{a}}(A)=-\infty$.)

$\left({ }^{3}\right)$ Note that the adopted convention is that of a Banach space duality, rather than the Hilbert space duality, hence the absence of the complex conjugation of the variable $s$. 
Corollary 3.2. Fix $p \in \mathbb{R}$ and consider $A_{s}^{T}: \mathrm{h}^{p} \rightarrow \mathrm{h}^{p}$. If $s=\sigma+i t$ satisfies $\sigma>\sigma_{\mathrm{a}}(A)-p$, then $A_{s}^{T}$ may be approximated in norm with any accuracy by finitely supported operators $\left({ }^{4}\right)$.

Proof. Let $\left.A_{s}^{T}\right\rfloor_{N}$ be the finitely supported operator obtained from $A_{s}^{T}$ by setting all $a_{n}$ with $n>N$ to zero. Let $\left.C_{s}^{T}=A_{s}^{T}-A_{s}^{T}\right\rfloor_{N}$. By Proposition 3.1.

$$
\left\|C_{s}^{T}\right\| \leq C^{\#}(p+\sigma)=\sum_{n>N}\left|a_{n}\right| n^{-(p+\sigma)} \rightarrow 0 \quad \text { as } N \rightarrow \infty .
$$

This proves the claim.

Corollary 3.3. If $p+\sigma>1$, then $Z_{s}^{T}: \mathrm{h}^{p} \rightarrow \mathrm{h}^{p}$ is a linear homeomorphism, and $\left(Z_{s}^{T}\right)^{-1}=M_{s}^{T}$.

Proof. By virtue of Proposition 3.1, if $p+\sigma>1$, then both $Z_{s}^{T}$ and $M_{s}^{T}$ are bounded operators in $\mathrm{h}^{p}$. Since the corresponding matrices commute and satisfy $M_{s}^{T} Z_{s}^{T}=I$, we have $M_{s}^{T}=\left(Z_{s}^{T}\right)^{-1}$.

EXAMPLE. It is interesting to observe by an explicit example how strongly indeed the properties of an operator of type $A_{s}^{T}$ depend on the space $\mathrm{h}^{p}$ in which it is considered. To this end consider $A=[(1,-1,0,0,0, \ldots)]$. Since $A(s)=1-2^{-s}$, so that $B(s)=A(s)^{-1}=1+2^{-s}+4^{-s}+8^{-s}+\cdots$, we conclude that $B_{s}$ is the formal inverse of $A_{s}$, and also $B_{s}^{T}$ is the formal inverse of $A_{s}^{T}$. Now, consider $A_{-1}^{T}$ (i.e. here $s=-1$ ), and let us examine the condition $A_{-1}^{T} f=0$ on $f$, which is equivalent to the following system of equations:

$$
\begin{aligned}
& f(1)-2 f(2)=0, \\
& f(2)-2 f(4)=0, \\
& f(3)-2 f(6)=0, \\
& f(4)-2 f(8)=0, \text { etc. }
\end{aligned}
$$

It is easy to find a nontrivial solution. Indeed, let $f$ be the sequence defined by $f(n)=1 / n$ when $n$ is an integer power of 2 , and $f(n)=0$ otherwise. Clearly, $A_{-1}^{T} f=0$. Moreover, $f \in \mathrm{h}^{p}$ for all $p<1$. Thus $\operatorname{ker} A_{-1}^{T}$ is nontrivial when $A_{-1}^{T}$ is considered in $\mathrm{h}^{p}$ with $p<1$. On the other hand, by virtue of Proposition 3.1, $B_{-1}^{T}: \mathrm{h}^{p} \rightarrow \mathrm{h}^{p}$ is a bounded operator whenever $p>1$ (because $\sigma_{\mathrm{a}}(B)=0$ ), and is the inverse of $A_{-1}^{T}$. Thus ker $A_{-1}^{T}=\{0\}$ when $A_{-1}^{T}$ is considered in $\mathrm{h}^{p}$ with $p>1$.

REMARK. A study on a related topic of Dirichlet multipliers in a certain Hilbert space may be found in [11].

$\left({ }^{4}\right)$ Note that finitely supported matrices are not finite rank. In fact, a nontrivial Dirichlet matrix is not even Hilbert-Schmidt, e.g. the Hilbert-Schmidt norm of $A: \mathrm{h}^{0} \rightarrow \mathrm{h}^{0}$ is equal to $\left(\sum_{m} \sum_{n}\left|a_{n}\right|^{2}\right)^{1 / 2}=\infty$. 
3.2. $Z: \ell_{1} \rightarrow \ell_{\infty}$ and $Z^{T}: \ell_{1} \rightarrow c_{0}$ are bounded. In this section we demonstrate that the action of $Z$ and $Z^{T}$ on $\ell_{1}$ defines continuous operators, provided one considers suitable topologies on the target spaces. The result that follows highlights the target spaces that seem natural for these operators. Below, ran $T$ denotes the range of an operator $T$.

THEOREM 3.1. The operators defined by the action of $Z$ and $Z^{T}$ on $\ell_{1}$ column vectors have the following properties:

(i) $Z: \ell_{1} \rightarrow \ell_{\infty}$ is bounded with $\|Z\|=1$ and $\operatorname{ker} Z=\{0\}$.

(ii) $\operatorname{ran} Z \cap c_{0}=\{0\}$.

(iii) $Z^{T}: \ell_{1} \rightarrow c_{0}$ is bounded with $\left\|Z^{T}\right\|=1$. Moreover, $\left(Z^{T}\right)^{*}=Z$. Also, $\operatorname{ran} Z^{T}$ is dense in $c_{0}$.

(iv) $\operatorname{ker} Z^{T}=\{0\}$. Equivalently, if $f \in \ell_{1}$ is such that $\sum_{k=1}^{\infty} f(n k)=0$ for all $n \in \mathbb{N}$, then $f=0$.

Proof. (i) In order to verify that $Z: \ell_{1} \rightarrow \ell_{\infty}$ is bounded, let $u \in \ell_{1}$, and let $g=Z u$. We have

$$
g(n)=\sum_{d \mid n} u(d), \quad \text { so } \quad|g(n)| \leq \sum_{d \mid n}|u(d)| \leq\|u\|_{1} \text { for all } n,
$$

i.e. $\|g\|_{\infty} \leq\|u\|_{1}$. Note that $\left\|Z \delta_{1}\right\|_{\infty}=\left\|\delta_{1}\right\|_{1}=1$. We conclude that $\|Z\|=1$.

In order to prove ker $Z=\{0\}$ let $g=Z u$ and assume $g(n)=0$ for all $n$. We use induction to show that $u(n)=0$ for all $n$. First, $u(1)=g(1)=0$. Next, assume that $0=u(1)=u(2)=\cdots=u(n-1)$. It follows that $0=g(n)=\sum_{d \mid n} u(d)=u(n)$, which completes the argument.

(ii) Let $f \in \ell_{1}$ and assume $g=Z f \in c_{0}$, i.e. $\lim _{n \rightarrow \infty} g(n)=0$. It suffices to demonstrate that $f=0$. We proceed by induction. Let $p$ denote primes. Since $g(p)=f(1)+f(p) \rightarrow f(1)$ as $p \rightarrow \infty$ over the set of primes, we have $f(1)=0$. Suppose that $f(k)=0$ for all $k=1, \ldots, n-1$. Let $n=p_{1}^{a_{1}} \ldots p_{k}^{a_{k}}$ be the prime decomposition of $n$. Assuming $p>\max \left\{p_{i}: i=1, \ldots, k\right\}$, we have

$$
g(n p)=\sum_{d \mid n}(f(d)+f(d p))=f(n)+\sum_{d \mid n} f(d p) \rightarrow f(n)
$$

(as $p \rightarrow \infty$ over the primes). Therefore $f(n)=0$ for all $n$.

(iii) For $f \in \ell_{1}$, let $g=Z^{T} f$, i.e. $g(n)=\sum_{k=1}^{\infty} f(n k)$. Thus,

$$
|g(n)| \leq \sum_{k=1}^{\infty}|f(n k)| \leq \sum_{k=n}^{\infty}|f(k)| .
$$

Since the sum on the right-hand side approaches 0 as $n \rightarrow \infty$, we conclude $g \in c_{0}$. Moreover, $\sup _{n}|g(n)| \leq \sum_{k=1}^{\infty}|f(k)|=\|f\|_{1}$, so $\left\|Z^{T}\right\| \leq 1$. However, if $f \geq 0$, then $g(1)=\|f\|_{1}$, so in fact $\left\|Z^{T}\right\|=1$. 
Next, recall that $c_{0}^{*}=\ell_{1}$ and $\ell_{1}^{*}=\ell_{\infty}$. Also, for $f \in c_{0}, g \in \ell_{1}$, and $h \in \ell_{\infty}$,

$$
g[f]=\langle f, g\rangle=\sum_{n=1}^{\infty} f(n) g(n) \quad \text { and } \quad h[g]=\langle g, h\rangle=\sum_{n=1}^{\infty} g(n) h(n) .
$$

Thus, for $u \in \ell_{1}$,

$$
\left\langle Z^{T} g, u\right\rangle=\sum_{n=1}^{\infty} \sum_{k=1}^{\infty} g(n k) u(n)=\sum_{m=1}^{\infty} g(m) \sum_{d \mid m} u(d)=\langle g, Z u\rangle,
$$

and so $Z=\left(Z^{T}\right)^{*}$. In addition, it follows that $\left(\operatorname{ran} Z^{T}\right)^{\perp}=\operatorname{ker}\left(Z^{T}\right)^{*}=$ $\operatorname{ker} Z=\{0\}$, which means that $\operatorname{ran} Z^{T}$ is dense in $c_{0}$ (see e.g. [5, p. 81 and p. 168]).

(iv) Suppose that $f \in \ell_{1}$ is such that $\sum_{k=1}^{\infty} f(n k)=0$ for all $n \in \mathbb{N}$. We will demonstrate that $f=0$. Denote by $\nu$ the measure on all subsets $A \subset \mathbb{N}$ defined by $\nu(A)=\sum_{n \in A} f(n)$. For a bounded function $h: \mathbb{N} \rightarrow \mathbb{R}$ we set $\int h d \nu=\sum_{n=1}^{\infty} h(n) f(n)$. In what follows we will use a version of the Dominated Convergence Theorem: if $h_{k}: \mathbb{N} \rightarrow \mathbb{R}$ is a uniformly bounded sequence of functions such that $\lim _{k \rightarrow \infty} h_{k}=h$, then $\lim _{k \rightarrow \infty} \int h_{k} d \nu=\int h d \nu$.

Next, let $I_{A}$ be the indicator function of $A \subset \mathbb{N}$. Denote $A_{k}=\{k n: n \in \mathbb{N}\}$. By assumption $\int I_{A_{k}} d \nu=\nu\left(A_{k}\right)=0$ for all $k \in \mathbb{N}$. Define

$$
h_{k}=\prod_{p \leq k}\left(I_{A_{1}}-I_{A_{p}}\right) \quad \text { (product over primes not exceeding } k \text { ) }
$$

It is seen by inspection that

$$
h_{k}=\sum_{n \in B_{k}} \mu(n) I_{A_{n}},
$$

where $B_{k}$ is the set of integers whose prime factors do not exceed $k$ and are mutually different. Since the sum is finite, $\int h_{k} d \nu=0$ for all $k$. At the same time $\lim _{k \rightarrow \infty} h_{k}=I_{\{1\}}$ as well as $0 \leq h_{k}(n) \leq 1$ for all $n \in \mathbb{N}$. Hence, $0=\int I_{\{1\}} d \nu=f(1)$. Applying the same arguments to the sequence $\tilde{f}(n)=f(k n)$ where $k$ is an arbitrary integer, we obtain $\tilde{f}(1)=f(k)=0$. This completes the proof $\left({ }^{5}\right)$.

REMARK 1. Since $Z=\left(Z^{T}\right)^{*}$, we have

$$
\{0\}=\operatorname{ker} Z^{T}={ }^{\perp}(\operatorname{ran} Z)=(\operatorname{ran} Z)^{\perp} \cap \ell_{1}
$$

(see e.g. [5, p. 168]). In other words, $\operatorname{ran} Z$ is weak*-dense in $\ell_{\infty}$. Also, the well known fact $\ell_{\infty}^{*} \neq \ell_{1}$ implies that $Z^{*}$ is not represented by $Z^{T}$. Note

$\left({ }^{5}\right)$ Alternatively, the fact that $\operatorname{ker} Z^{T}=\{0\}$ may be deduced from the Dynkin $\pi-\lambda$ Theorem. 
that this can be easily deduced from Theorem 3.1 without a reference to the classical result.

REMARK 2. The fact that $Z$ is not surjective, which is weaker than statement (ii) in Theorem 3.1, can be proved by a general argument. Indeed, by virtue of Pitt's compactness theorem every bounded operator from $c_{0}$ to $l_{1}$ is compact (see e.g. [1, 7]). In particular $Z^{T}: l_{1} \rightarrow c_{0}$ has no inverse. Further, recall that an operator is invertible if and only if its adjoint is invertible (see e.g. [5, Proposition 1.9, p. 169]). Therefore, $Z: l_{1} \rightarrow l_{\infty}$ has no inverse either. Since, as we have seen, $Z$ is injective, it follows from the open mapping theorem that it is not surjective.

Remark 3. It is known that $\ell_{1}$ with the Dirichlet product $\left({ }^{6}\right)$ is a unital commutative Banach algebra. Indeed, using the notation adopted above, we have $g * f=G f$, where $G=[(g(1), g(2), g(3), \ldots)]$ for all $f, g \in \ell_{1}$. Next, observe that every column of $G$ has the same $\ell_{1}$ norm (namely, $\|g\|=\|g\|_{1}$ ), which implies $G: \ell_{1} \rightarrow \ell_{1}$ with $\|G\| \leq\|g\|$; for a proof see [12, Example 2.3, Chapter III]. It follows that $\|g * f\| \leq\|g\| \cdot\|f\|$, and hence $\left(\ell_{1}, *\right)$ is indeed a Banach algebra. In light of this it is natural to ask if $Z^{T}: \ell_{1} \rightarrow c_{0}$ preserves the Dirichlet multiplication. The answer is negative. Indeed, it is easily seen that $\delta_{2} * \delta_{6}=\delta_{12}$, while

$$
Z^{T} \delta_{12}=\sum_{d \mid 12} \delta_{d} \neq \sum_{d \mid 2} \delta_{d} * \sum_{d \mid 6} \delta_{d}=Z^{T} \delta_{2} * Z^{T} \delta_{6} .
$$

However, interestingly, if $\operatorname{GCD}(k, l)=1$, then

$$
Z^{T}\left[\delta_{k} * \delta_{l}\right]=\sum_{d \mid k l} \delta_{d}=\sum_{d \mid k} \delta_{d} * \sum_{d \mid l} \delta_{d}=Z^{T} \delta_{k} * Z^{T} \delta_{l} .
$$

3.3. $Z^{T}: \ell_{1, \mathrm{~m}} \rightarrow \ell_{1, \mathrm{~m}}$ and $Z: \ell_{\infty, \mathrm{m}^{-1}} \rightarrow \ell_{\infty, \mathrm{m}^{-1}}$ are bounded. It is interesting whether $Z$ and $Z^{T}$ give rise to operators in a (single) Banach space. An affirmative answer is supplied by a weighted $\ell_{1}$. Indeed, let us introduce a weight function $\mathrm{m}: \mathbb{N} \rightarrow \mathbb{N}$ via recurrence as follows:

$$
\mathrm{m}(1)=1, \quad \mathrm{~m}(n)=\sum_{d \mid n, d<n} \mathrm{~m}(d) \quad(n>1) .
$$

Note the obvious identity

$$
\sum_{k \mid n} \mathrm{~m}(k)=2 \mathrm{~m}(n)
$$

Next, it is easily seen that

$$
\ell_{1, \mathrm{~m}}=\left\{f: \mathbb{N} \rightarrow \mathbb{C}:\|f\|_{1, \mathrm{~m}}:=\sum_{n \in \mathbb{N}}|f(n)| \mathrm{m}(n)<\infty\right\}
$$

$\left({ }^{6}\right)$ See footnote $\left({ }^{1}\right)$. 
is a Banach space. In addition

$$
\ell_{\infty, \mathrm{m}^{-1}}=\left\{f: \mathbb{N} \rightarrow \mathbb{C}:\|f\|_{\infty, \mathrm{m}^{-1}}:=\sup _{n \in \mathbb{N}} \frac{|f(n)|}{\mathrm{m}(n)}<\infty\right\}
$$

is the dual of $\ell_{1, \mathrm{~m}}$. Moreover, we have the following:

Proposition 3.2. The action of the matrix $Z^{T}$ (resp. $Z$ ) defines an operator in $\ell_{1, \mathrm{~m}}\left(\right.$ resp. $\left.\ell_{\infty, \mathrm{m}^{-1}}\right)$ with the following properties:

(i) $Z^{T}: \ell_{1, \mathrm{~m}} \rightarrow \ell_{1, \mathrm{~m}}$ is bounded with $\left\|Z^{T}\right\| \leq 2$.

(ii) $Z: \ell_{\infty, \mathrm{m}^{-1}} \rightarrow \ell_{\infty, \mathrm{m}^{-1}}$ is bounded with $\|Z\| \leq 2$.

Proof. It is easily seen that $Z: \ell_{\infty, \mathrm{m}^{-1}} \rightarrow \ell_{\infty, \mathrm{m}^{-1}}$ is the adjoint of $Z^{T}: \ell_{1, \mathrm{~m}} \rightarrow \ell_{1, \mathrm{~m}}$. Therefore, the second statement is a consequence of the first (see e.g. [5]).

In order to prove the first statement let $f \in \ell_{1, \mathrm{~m}}$ and $g=Z^{T}[f]$, i.e.

$$
g(n)=\sum_{k=1}^{\infty} f(n k)
$$

It suffices to show $\|g\|_{1, \mathrm{~m}} \leq 2\|f\|_{1, \mathrm{~m}}$. Taking into account (5) we observe

$$
\begin{aligned}
\|g\|_{1, \mathrm{~m}} & =\sum_{n \in \mathbb{N}}|g(n)| \mathrm{m}(n) \\
& \leq \mathrm{m}(1) \sum_{k=1}^{\infty}|f(k)|+\mathrm{m}(2) \sum_{k=1}^{\infty}|f(2 k)|+\mathrm{m}(3) \sum_{k=1}^{\infty}|f(3 k)|+\cdots \\
& =\sum_{n=1}^{\infty}|f(n)| \sum_{k \mid n} \mathrm{~m}(k)=2 \sum_{n=1}^{\infty}|f(n)| \mathrm{m}(n)=2\|f\|_{1, \mathrm{~m}} .
\end{aligned}
$$

This completes the proof.

REMARK. In the above proof the norm of $Z^{T}$ is determined by identity (5). One might redefine $\mathrm{m}$ by setting $\mathrm{m}(n)=C \sum_{d \mid n, d<n} \mathrm{~m}(d)(n>1)$ for any positive real $C$. In the consistently redefined $\ell_{1, \mathrm{~m}}$ one would then have $\left\|Z^{T}\right\| \leq 1+C$.

Acknowledgments. I am grateful for the advice I have received from the editors and referees of the Bulletin PAN with regard to some of the content of this work as well as the style of presentation and target audience. I also acknowledge the support of the Canadian Foundation for Innovation, grant LOF \# 22117.

\section{References}

[1] F. Albiac and N. J. Kalton, Topics in Banach Space Theory, Grad. Texts in Math. 233, Springer, 2006.

[2] T. M. Apostol, Introduction to Analytic Number Theory, Springer, New York, 1976. 
[3] P. T. Bateman and H. G. Diamond, Analytic Number Theory, An Introductory Course, World Sci., 2009.

[4] A. Beurling, The Collected Works of Arne Beurling, Vol. 2: Harmonic Analysis, Birkhäuser Boston, 1989, 378-380.

[5] J. B. Conway, A Course in Functional Analysis, 2nd ed., Grad. Texts in Math. 96, Springer, 1990.

[6] I. Daubechies, Ten Lectures on Wavelets, CBMS-NSF Reg. Conf. Ser. Appl. Math. 61, SIAM, 1992.

[7] S. Delpech, A short proof of Pitt's compactness theorem, Proc. Amer. Math. Soc. 137 (2009), 1371-1372.

[8] H. M. Edwards, Riemann's Zeta Function, Academic Press, New York, 1974.

[9] D. N. Green, Generating nonorthogonal bases for signal representations, Circuits Systems Signal Process. 3 (1984), 447-475.

[10] D. N. Green and S. C. Bass, Representing periodic waveforms with nonorthogonal basis functions, IEEE Trans. Circuits Systems 31 (1984), 518-584.

[11] H. Hedenmalm, P. Lindqvist, and K. Seip, A Hilbert space of Dirichlet series and systems of dilated functions in $L^{2}(0,1)$, Duke Math. J. 86 (1997), 1-37.

[12] T. Kato, Perturbation Theory for Linear Operators, Springer, 1980.

[13] H. L. Montgomery, Ten Lectures on the Interface Between Analytic Number Theory and Harmonic Analysis, CBMS Reg. Conf. Ser. Math. 84, Amer. Math. Soc., 1994.

[14] A. Sowa, A fast-transform basis with hysteretic features, in: Proc. 24th Canadian Conf. Electrical and Computer Engineering, 2011, 253-257.

[15] A. Sowa, On an eigenvalue problem with a reciprocal-linear term, Waves Random Complex Media 22 (2012), 186-206.

[16] A. Sowa, Signals generated in memristive circuits, Nanoscale Systems Math. Modeling Theory Appl. 1 (2012), 48-57.

[17] A. Sowa, Factorizing matrices by Dirichlet multiplication, Linear Algebra Appl. 438 (2013), 2385-2393.

[18] A. Sowa, The Dirichlet ring and unconditional bases in $L_{2}[0,2 \pi]$, Funct. Anal. Appl. 47 (2013), 227-232.

[19] A. Sowa, Riemann's zeta function and the broadband structure of pure harmonics, arXiv:1603.03667 [math.GM] (2016).

[20] A. Sowa, Encoding spatial data into quantum observables, arXiv:1609.01712 [quant$\mathrm{ph}$ ] (2016).

[21] P. Wojtaszczyk, A Mathematical Introduction to Wavelets, London Math. Soc. Student Texts 37, Cambridge Univ. Press, 1997.

[22] R. M. Young, An Introduction to Nonharmonic Fourier Series, rev. ed., Academic Press, San Diego, 2001.

Artur Sowa

Department of Mathematics and Statistics

University of Saskatchewan

106 Wiggins Road

Saskatoon, SK S7N 5E6, Canada

E-mail: sowa@math.usask.ca 